\title{
A small internal antisense RNA (aftsH) of all3642 (ftsH) in Anabaena sp. PCC 7120
}

\author{
GONG YangMin \& XU XuDong* \\ State Key Laboratory of Freshwater Ecology and Biotechnology, Institute of Hydrobiology, Chinese Academy of Sciences, Wuhan 430072, China
}

Received September 12, 2011; accepted November 11, 2011

\begin{abstract}
In bacteria, FtsH proteases are involved in quality maintenance of membrane proteins and cellular regulation at the level of protein stability. Among the 4 predicted ftsH genes in the cyanobacterium Anabaena sp. PCC 7120, namely alr1262, all3642, all4776 and all4936, at least the first two showed reduced transcription in specialized $\mathrm{N}_{2}$-fixing cells called heterocysts. In contrast, the transcription of a small internal antisense RNA (aftsH) of all3642 was up-regulated in heterocysts. Overexpression of the antisense RNA suppressed the expression of all3642, alr1262 and all4776. Control of promoter and modulation by antisense RNA may both contribute to gene regulation in heterocysts of filamentous cyanobacteria.
\end{abstract}

antisense RNA, Anabaena 7120, fts $\mathrm{H}$ genes

Citation: Gong Y M, Xu X D. A small internal antisense RNA (aftsH) of all3642 (ftsH) in Anabaena sp. PCC 7120. Chin Sci Bull, 2012, 57: 756-761, doi: 10.1007/s11434-011-4929-6

Cyanobacteria are a group of prokaryotes that perform oxygenic photosynthesis [1]. Anabaena/Nostoc sp. PCC 7120 (Anabaena 7120) can produce specialized $\mathrm{N}_{2}$-fixing cells, called heterocysts, along their uniseriate filaments upon nitrogen stepdown. Unlike vegetative cells, heterocysts possess a secondary envelope consisting of the outside polysaccharide layer and the inner glycolipid layer, perform $\mathrm{N}_{2}$-fixation and show significantly enhanced respiratory activity, but no longer perform oxygenic photosynthesis and cell division [2]. During the development process from vegetative cells to heterocysts, a large number of genes are upor down-regulated [3].

FtsH is a transmembrane protein with a main cytosolic region consisting of AAA-ATPase and $\mathrm{Zn}^{2+}$-metalloprotease domains [4]. It is involved in cellular regulation at the level of protein stability and quality maintenance of membrane proteins. In the unicellular cyanobacterium Synechocystis sp. PCC 6803 (Synechocystis 6803), one of the ftsH genes, slr0228, has been extensively studied. The slr0228encoded FtsH was found to be required for quality control

*Corresponding author (email: xux@ihb.ac.cn) of PSII [5], induction of inorganic carbon acquisition complex [6], and involved in osmoregulation by degrading GgpS [7]. In Anabaena 7120, no studies on FtsH-encoding genes have been reported.

Noncoding RNAs, especially antisense RNAs, have been found in different groups of cyanobacteria [8-13], and the regulatory effects of antisense RNAs have been shown in at least 2 species. In Synechocystis 6803, over-expression of an internal antisense RNA, isrR, significantly inhibited the expression of the target gene isiA [9]. In Anabaena 7120, the furA gene (all1691) is not only transcribed in its own direction, but also co-transcribed with alr1690 in reverse direction, and interruption of alr1690 increased the expression level of FurA protein [12]. Also, a non-coding RNA of about $60 \mathrm{nt}$ specifically transcribed in heterocysts has been identified in Anabaena 7120 [14], but its role in gene regulation remains to be elucidated. More recently, some antisense RNAs of genes relating to heterocyst differentiation were identified by mRNA sequencing [10]. In this study, an internal antisense RNA of a gene encoding FtsH was identified in Anabaena 7120. The antisense RNA showed inhibitory effects on $\mathrm{ftsH}$ genes, two of which were down-regulated in heterocysts. 


\section{Materials and methods}

\subsection{Strains and culture conditions}

Anabaena sp. strain PCC 7120 and its derivatives were cultured in BG11 as previously described [15]. Conjugative transfer of plasmids into Anabaena 7120 was performed as described by Elhai and Wolk [16]. Anabaena strains are listed in Table 1.

\subsection{Plasmid construction}

Molecular manipulations were performed using standard methods. Tool enzymes were used according to the manufacturers' instructions. PCR fragments were cloned in the T-vector pMD18-T (TaKaRa, Japan) and confirmed by sequencing. The plasmids and primers used are listed in Table 1. The plasmids pHB1213 containing $\mathrm{P}_{\text {all3642- }} g f$, PHB1295 containing $\mathrm{P}_{\text {alr 1261- }}$ gfp and pHB1296 containing $\mathrm{P}_{\text {all4776- }}$ gfp were used for analyses of transcription from $\mathrm{ftsH}$ promoters. The plasmid pHB3143 carrying $\mathrm{P}_{r b c L}$-aftsHomega was used to overexpress aftsH, pHB3891 carrying $\mathrm{P}_{r b c L^{-}}$-omega was used as the empty control, pHB3009 was used to produce the conserved portion of FtsH in $E$. coli.

\subsection{Northern blot analyses}

Northern blot hybridization was performed according to
Zhang et al. [18]. Primers used to generate the DIG-labeled probes are listed in Table 1.

\subsection{Western blot analyses}

Western blot analysis was performed as described by $\mathrm{He}$ and $\mathrm{Xu}$ [20]. FtsH proteins were detected with the rabbit antiserum raised against a conserved portion of FtsH.

\subsection{Rapid amplification of cDNA ends}

5' RACE was performed as described by Zhang et al. [18] using nested PCR. cDNA tailed with SMART II A Oligonucleotide was synthesized using a SMART RACE cDNA amplification kit (ClonTech, TaKaRa Bio) and random primers (Promega). The universal primer A mix provided with the kit and a gene-specific primer (aftsH5RACE-1) were used in the first-round PCR, and the nested universal primer $A$ and the second gene-specific primer (aftsH5RACE-2) were used in the 2nd-round PCR.

3' RACE was performed as described by Argaman et al. [21] with minor modifications. Total RNA was ligated with $3^{\prime}$ adapter ( $3^{\prime}$ linker), reverse-transcribed with adapterspecific primer ( $3^{\prime}$ linkerRTrev). The products of reverse transcription were used as the template for nested PCR using primer pairs aftsH3RACE-1/3' linkerPCRrev in the first- round PCR and aftsH3RACE-2/3' linkerPCRrev in the second-round PCR.

Table 1 Anabaena strains, plasmids and primers

\begin{tabular}{|c|c|c|}
\hline Strain, plasmid or primer & Derivation, relevant characteristics or sequence & Source or reference \\
\hline \multicolumn{3}{|l|}{ Anabaena strains } \\
\hline Anabaena sp. PCC 7120 & Wild type (WT) & $\mathrm{FACHB}^{\mathrm{a})}$ \\
\hline WT (pHB1835) & $\begin{array}{l}\mathrm{Sp}^{\mathrm{r}} \text {, pHB } 1835 \text { carrying chromosomal DNA bp } 4393690 \text { to } 4395128 \text { upstream of } g f p \text { in pHB912 } \\
\text { introduced into Anabaena } 7120\end{array}$ & [17]; this study \\
\hline WT (pHB2918) & $\begin{array}{l}\mathrm{Sp}^{\mathrm{r}} \text {, pHB2918 carrying chromosomal DNA bp } 4393694 \text { to } 4394934 \text { upstream of } g f p \text { in pHB912 } \\
\text { introduced into Anabaena } 7120\end{array}$ & This study \\
\hline WT (pHB3143) & 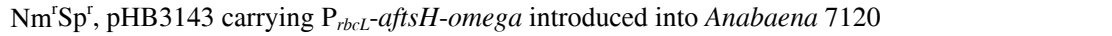 & This study \\
\hline WT (pHB3891) & $\mathrm{Nm}^{\mathrm{r}} \mathrm{Sp}^{\mathrm{r}}$, pHB3891 carrying $\mathrm{P}_{r b c L}$-omega (empty vector) introduced into Anabaena 7120 & This study \\
\hline \multicolumn{3}{|l|}{ Plasmids $^{\text {b) }}$} \\
\hline $\mathrm{pDC} 8$ & $\begin{array}{l}\mathrm{Km}^{\mathrm{r}} \text {, pDU1-based CAT (chloramphenicol acetyl transferase)-reporting vector (NCBI GenBank } \\
\text { accession number EU047752) }\end{array}$ & [18] \\
\hline pET21b & $\mathrm{Ap}^{\mathrm{r}}$, overexpression vector & Novagen \\
\hline pHB1071 & $\begin{array}{l}\mathrm{Ap}^{\mathrm{r}} \mathrm{Sp} \mathrm{p}^{\mathrm{r}} \text {, BamH I-cut pUC19, inserted into } B g l \mathrm{II}-\mathrm{cut} \mathrm{pHB} 912 \text {, to provide } S m a \mathrm{I}-S s e 8387 \text { I sites for } \\
\text { cloning of DNA fragments upstream of } g f p\end{array}$ & [18] \\
\hline pHB1192 & $\begin{array}{l}\left.\text { Ap }^{\mathrm{r}} \text {, PCR fragment (Anabaena chromosomal bp } 4395267 \text { to } 4396630\right) \text { generated with primers } \\
\text { ftsH3642-1 and ftsH3642-2, cloned into pMD18-T }\end{array}$ & This study \\
\hline pHB1193 & $\begin{array}{l}\left.\text { Ap }^{\mathrm{r}} \text {, PCR fragment (Anabaena chromosomal bp } 1493544 \text { to } 1495668\right) \text { generated with primers } \\
\text { ftsH1261-1 and ftsH1261-2, cloned into pMD18-T }\end{array}$ & This study \\
\hline pHB1245 & $\begin{array}{l}\mathrm{Ap}^{\mathrm{r}} \text {, PCR fragment (Anabaena chromosomal bp } 5691135 \text { to } 5691531 \text { ) generated with primers } \\
\text { ftsH4776-1 and ftsH4776-2, cloned into pMD18-T }\end{array}$ & This study \\
\hline pHB1213 & $\begin{array}{l}\mathrm{Sp}^{\mathrm{r}} \text {, the DNA fragment excised from pHB1192 with Sma I and Pst } \mathrm{I} \text {, cloned into Sma I- and } \\
\text { Sse8387 I-cut pHB1071, for expression of } g f p \text { from } \mathrm{P}_{\text {all3642 }} \text { in Anabaena } 7120\end{array}$ & This study \\
\hline pHB1295 & $\begin{array}{l}\mathrm{Sp}^{\mathrm{r}} \text {, the DNA fragment excised from pHB1193 with Sma I and Pst I, cloned into Sma I- and } \\
\text { Sse8387 I-cut pHB1071, for expression of } g f p \text { from } \mathrm{P}_{\text {alr } 2261} \text { in Anabaena } 7120\end{array}$ & This study \\
\hline pHB1296 & $\begin{array}{l}\mathrm{Sp}^{\mathrm{r}} \text {, the DNA fragment excised from pHB1245 with Sma I and Pst } \mathrm{I} \text {, cloned into Sma I- and } \\
\text { Sse8387 I-cut pHB1071, for expression of } g f p \text { from } \mathrm{P}_{\text {all }} \text { (4776 in Anabaena } 7120\end{array}$ & This study \\
\hline
\end{tabular}


(Continued)

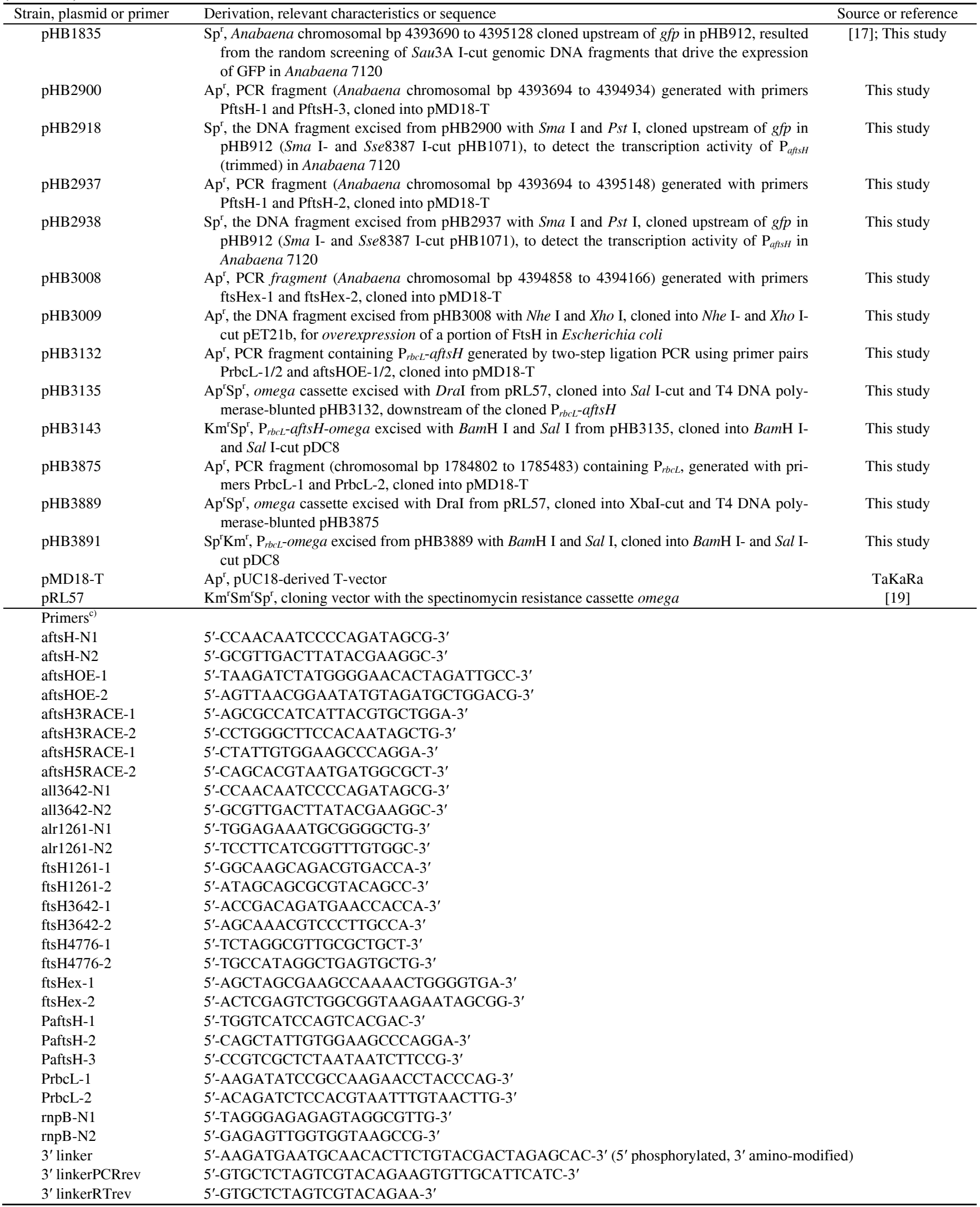

a) FACHB, Freshwater Algal Culture Collection of the Institute of Hydrobiology, Chinese Academy of Sciences. b) Ap, ampicillin; Nm, neomycin; Km, kanamycin; Sm, streptomycin; Sp, spectinomycin; unless stated otherwise, the template for PCR reactions was Anabaena genomic DNA. c) N1 and N2 indicate primers used to generate probes for Northern blot hybridization; RT1 and RT2 indicate primers used in RT-PCR. 


\section{Results and discussion}

\subsection{A small internal antisense RNA (aftsH) of all3642 (ftsH)}

In a project using $g f p$ (green-fluorescence protein) as the reporter gene to identify regulated promoters in heterocysts or developing proheterocysts in the filamentous cyanobacterium Anabaena 7120, we cloned many genomic DNA fragments that showed up- or down-regulated promoter activity in proheterocysts or heterocysts [17]. Some DNA fragments cloned upstream of the $g f p$ reporter gene indicated that transcription started in an antisense direction within an ORF. One such fragment (Anabaena 7120 chromosomal 4393690-4395128 bp, pHB1835 in Table 1) was located within all3642 (chromosomal 4395350-4393464 bp), a gene predicted to encode the FtsH protease. Transcription from this fragment or a PCR fragment located between chromosomal 4393694 and 4395148 bp (pHB2938 in Table 1) showed an up-regulation in heterocysts $\left(\mathrm{P}_{\text {afs } H}\right.$ in Figure 1 and data not shown), while trimming of chromosomal 4394935-4395148 bp from this fragment (pHB2918 in Table 1) greatly reduced the transcription activity in whole filaments and eliminated the up-regulation in heterocysts $\left(\mathrm{P}_{a f t s H}\right.$ trimmed in Figure 1). A Northern blot probed with an internal fragment, chromosomal 4394984-4395175 bp, showed a transcript of $\sim 1.8 \mathrm{~kb}$ and a much smaller RNA of $\sim 0.2 \mathrm{~kb}$ (Figure 2(c)). The larger transcript was the mRNA of all3642, whereas the smaller transcript was an internal anti- sense RNA. 5'- and 3'-RACEs mapped the small RNA, denoted afts $H$, between chromosomal 4394967 and 4395199 bp in opposite orientation to all3642 (Figure 2(a); Figure 3).

\subsection{Transcription of $f t s H$ genes in heterocysts}

In Anabaena 7120, 3 other genes, alr1261, all4776 and all4936 are predicted to encode FtsH proteases, in addition to all3642. At the nucleic acid level, all3642 shows $79 \%$, $86 \%$ and $51 \%$ identities with alr1261, all4776 and all4936, respectively (Figure S1). At amino acid level, All3642 shares 63\%, 74\% and 48\% identities with Alr1261, All4776 and All4936, respectively (Figure S2). All the 4 predicted FtsH proteases are composed of the N-terminal transmembrane segments and the $\mathrm{C}$-terminal regions containing the protease and $\mathrm{AAA}^{+}$domains, of which the $\mathrm{AAA}^{+}$domain consists of conserved Walker A and Walker B motifs (Figure S2) [22].

We examined the expression of $4 \mathrm{ftsH}$ genes using $g f p$ as the reporter gene. Transcription from $\mathrm{P}_{\text {all3642 }}$ and $\mathrm{P}_{\text {alr } 1261}$ was down-regulated in heterocysts, while that from $\mathrm{P}_{\text {all4776 }}$ was not (Figure 1). No GFP fluorescence could be found in either vegetative cells or heterocysts of the strain carrying

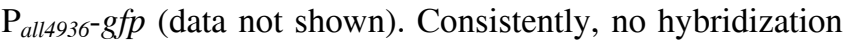
signal was detectable in Northern blot analysis of the expression of all4936 (data not shown). The differential expression pattern of $\mathrm{fts} H$ genes indicated that four $\mathrm{ftsH}$ genes in Anabaena 7120 might be involved in different cellular processes.
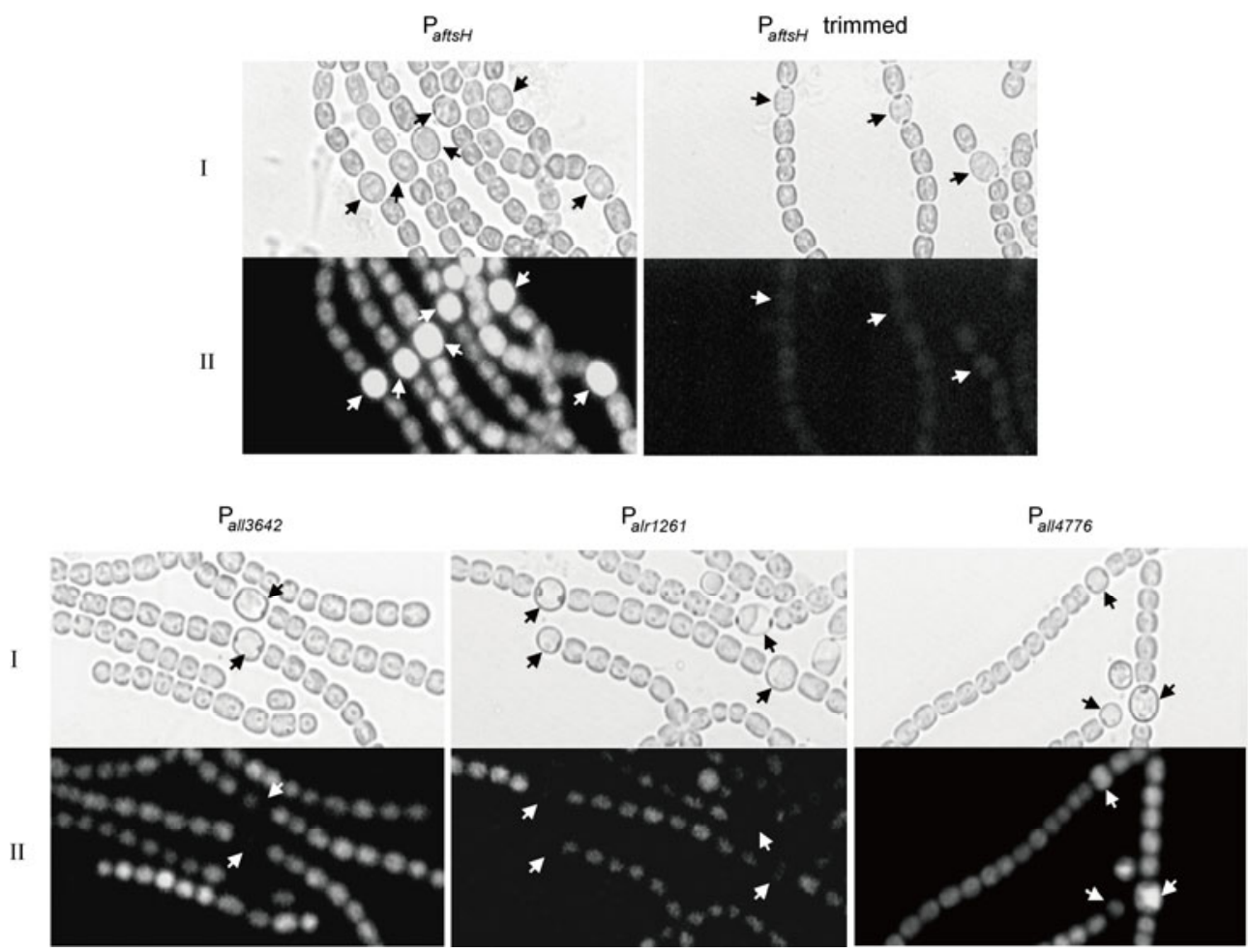

Figure 1 Bright field (I) and GFP fluorescence (II) images of Anabaena 7120 bearing $\mathrm{P}_{a f s H^{-}} g f p$ (pHB1835), $\mathrm{P}_{a f t s H}$ (trimmed)-gfp (pHB2918), $\mathrm{P}_{a l l 3642^{-}} g f p$ (pHB1213), $\mathrm{P}_{\text {alrl261- }}$ ffp (pHB1295) or $\mathrm{P}_{\text {all4776- }}$ gfp (pHB1296) after nitrogen step-down for $24 \mathrm{~h}$. Arrows point to heterocysts or proheterocysts. 
(a)

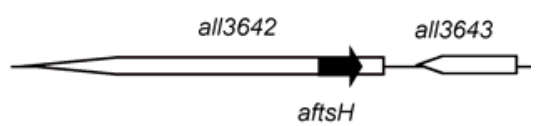

(b)

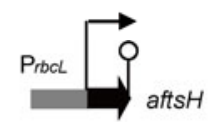

(d)
all3642

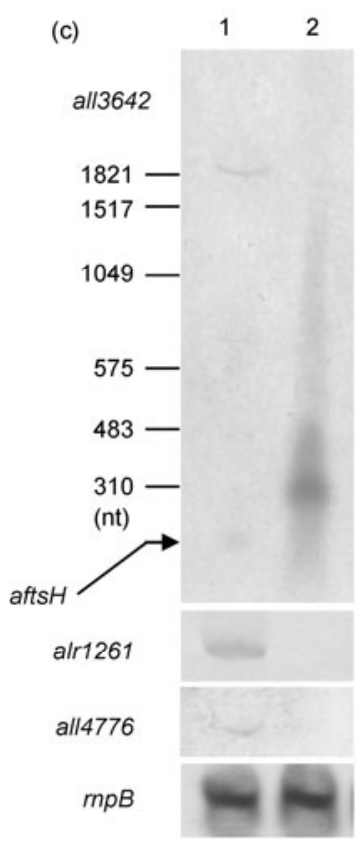

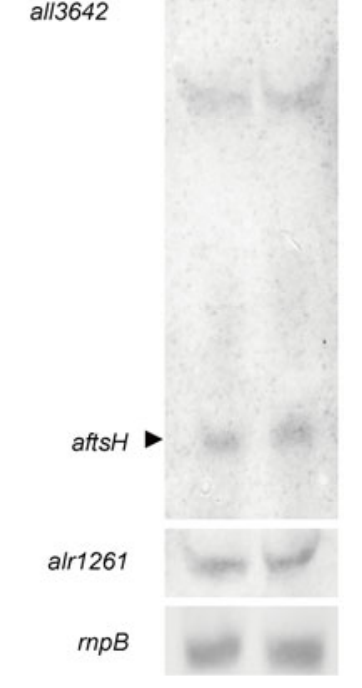

(e)

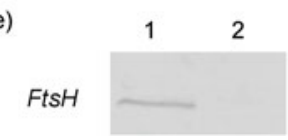

Figure 2 Inhibitory effect of afts $H$ on the expression of $f t s H$ genes in Anabaena 7120. (a) A schematic diagram showing all3642 and the position/orientation of the afts $H$-transcribed region. (b) A schematic diagram showing the clone for expressing aftsH from $\mathrm{P}_{r b c L}$ in Anabaena. The bent arrow indicates the start site of transcription; the stem-loop symbolizes the transcriptional terminator. (c) Northern blot analyses of $f t s H$ transcripts and aftsH in Anabaena 7120 without (lane 1) or with $\mathrm{P}_{r b c L}$-aftsH (lane 2). rnpB was used as the internal control. (d) Northern blot analyses of $f t s H$ transcripts and aftsH in Anabaena 7120 without (lane 1) or with pHB3891 (carrying $\mathrm{P}_{r b c L}$ but lacking $a f t s H$ ) as the empty vector control (lane 2). rnpB was used as the internal control. (e) Western blot detection of FtsH in Anabaena 7120 without (lane 1) or with $\mathrm{P}_{r b c}$-aftsH (lane 2).

\subsection{Down-regulation of $f t s H$ genes by overexpressed antisense RNA}

Due to the down-regulation of the $2 \mathrm{ftsH}$ genes in heterocysts, one may not see the effect of overexpression of the antisense RNA on their transcripts in heterocysts. Also, quantitative analysis of mRNA in heterocysts is hardly feasible. Extensive RNA degradation could have occurred before heterocysts are isolated from vegetative cells. Hence, we tested the effect of afts $H$ in vegetative cells. The antisense RNA was expressed from the promoter (chromosomal 1784801-1785483 bp) of $r b c L$, which encodes the large subunit of ribulose-1,5-bisphosphate carboxylase (WT (pHB3143) in Table 1; Figure 2(b)). $\mathrm{P}_{r b c L}$ is a strong promoter expressed in vegetative cells and down-regulated in heterocysts [23]. The DNA sequence of afts $H$ was fused to $\mathrm{P}_{r b c L} 19$ bp downstream of its transcriptional start point. The overexpressed antisense RNA greatly reduced the abundance of all3642, alr1261 and all4776 transcripts (Figure 2(c) in Anabaena 7120). The empty vector with $\mathrm{P}_{r b c L}$ but lacking aftsH showed no effect on the expression of alr1261 and all3642 (WT (pHB3891) in Table 1; Figure 2(d)).

The regulatory effect of afts $H$ was also detected at the protein level. A 231 -aa sequence, $73 \%$ to $96 \%$ identical to the 4 predicted FtsH proteins (Figure S2), was used to generate rabbit anti-FtsH antiserum. Western blot detection of FtsH showed a result consistent with the Northern blot detection (Figure 2(e)). The significantly reduced expression of fts $\mathrm{H}$ genes in Anabaena 7120, however, affected the growth under neither nitrogen-fixing nor nitrogen-replete conditions, suggesting that the low level of FtsH was sufficient to support the cellular processes in the cyanobacterium.

All3642 shows $80 \%$ identity to Slr0228 and $49 \%-66 \%$ identity to the other 3 predicted FtsH proteins in Synechocystis 6803 . Very probably, all3642 is required for quality control of PSII in Anabaena 7120 as slr0228 in Synechocystis 6803 [5]. Functions of the rest ftsH genes in Synechocystis CTTACGGTCGCTTTTTGGAATATGTAGATGCTGGACGTGTTACCAGCGTTGACTTATACGAAGGC
GAATGCCAGCGAAAAACCTTATACATCTACGACCTGCACAATGGTCGCAACTGAATATGCTTCCG
$\triangle$ TTP
GGCAGAACAGCTATTGTGGAAGCCCAGGATCAAGATATCGAAAACCGTGTCCAACGTTGGCGGGT
CCGTCTTGTCGATAACACCTTCGGGTCTAGTTCTATAGCTTTTGGCACAGGTTGCAACCGCCCA

GGATTTGCCAGTTAGCGCTCCTGAGTTAATCAGCAAACTTAAAGACAAGAAAGTAAGTTTTGATG CCTAAACGGTCAATCGCGAGGACTCAATTAGTCGTTTGAATTTCTGTTCTTTCATTCAAAACTAC

CTCATCCAGCACGTAATGATGGCGCTATCTGGGGATTGTTGGGCAATCTAGTGTTCCCCATCTTA GAGTAGGTCGTGCATTACTACCGCGATAGACCCCTAACAACCCGTTAGATCACAAGGGGTAGAAT TSP

Figure 3 The double-stranded sequence of the aftsH locus within all3642 (partial). Transcriptional start point (TSP) and transcriptional termination point (TTP) are boxed. The shaded region is the afts $H$-encoding sequence, and the underlined region is the sequence used as the probe for Northern blot analyses. 
and Anabaena, however, are not identified yet. As a cisencoded antisense RNA, aftsH may accelerate the downregulation of all3642 in developing heterocysts. Also, it may act in trans to down-regulate other ftsH genes. It is noteworthy that the corresponding regions of alr1261 and all4776 show only $43 \%$ and $30 \%$ identities to the afts $\mathrm{H}$ encoding sequence of all3642 (Figure S1). Apparently, the regulation of alr1261 and all4776 by afts $H$ requires only a partial match between the antisense RNA and mRNAs, which is consistent with the view point that antisense RNAs can mediate inhibition by forming partial duplexes with target mRNAs [24].

The authors are indebted to Dr. Wang Yu for technical assistances. This work was supported by the National Natural Science Foundation of China (30825003, 30770049).

1 Castenholz R W. Phylum B X. Cyanobacteria oxygenic photosynthetic bacteria. In: Boone D R, Castenholz R W, eds. Bergey's Manual of Systematic Bacteriology. 2nd ed. New York, Berlin, Heidelberg: Springer, 2001. 474-487

2 Wolk C P, Ernst A, Elhai J. Heterocyst metabolism and development. In: Bryant D A, ed. The Molecular Biology of Cyanobacteria. Dordrecht, NL: Kluwer Academic Publishers, 1994. 769-823

$3 \mathrm{Xu} \mathrm{X}$, Elhai J, Wolk C P. Transcriptional and developmental responses by Anabaena to deprivation of fixed nitrogen. In: Herrero T, Flores E, eds. Cyanobacteria: Molecular Biology, Genomics and Evolution. Norwich: Horizon Scientific Press, 2008. 383-422

4 Ito K, Akiyama Y. Cellular functions, mechanism of action, and regulation of FtsH protease. Annu Rev Microbiol, 2005, 59: 211-231

5 Komenda J, Barker M, Kuviková S, et al. The FtsH protease slr0228 is important for quality control of photosystem II in the thylakoid membrane of Synechocystis sp. PCC 6803. J Biol Chem, 2006, 281: 1145-1151

6 Zhang P, Sicora C I, Vorontsova N, et al. FtsH protease is required for induction of inorganic carbon acquisition complexes in Synechocystis sp. PCC 6803. Mol Microbiol, 2007, 65: 728-740

7 Stirnberg M, Fulda S, Huckauf J, et al. A membrane-bound FtsH protease is involved in osmoregulation in Synechocystis sp. PCC 6803: The compatible solute synthesizing enzyme GgpS is one of the targets for proteolysis. Mol Microbiol, 2007, 63: 86-102

8 Csiszàr K, Houmard J, Damerval T N, et al. Transcriptional analysis of the cyanobacterial $g v p A B C$ operon in differentiated cells: Occur- rence of an antisense RNA complementary to three overlapping transcripts. Gene, 1987, 60: 29-37

9 Dühring U, Axmann I M, Hess W R, et al. An internal antisense RNA regulates expression of the photosynthesis gene isiA. Proc Natl Acad Sci USA, 2006, 103: 7054-7058

10 Flaherty B L, Van Nieuwerburgh F, Head S R, et al. Directional RNA deep sequencing sheds new light on the transcriptional response of Anabaena sp. strain PCC 7120 to combined-nitrogen deprivation. BMC Genomics, 2011, 12: 332

11 Georg J, Vo B, Scholz I, et al. Evidence for a major role of antisense RNAs in cyanobacterial gene regulation. Mol Syst Biol, 2009, 5: 305

12 Hernández J A, Muro-Pastor A M, Flores E, et al. Identification of a furA cis antisense RNA in the cyanobacterium Anabaena sp. PCC 7120. J Mol Biol, 2005, 355: 325-334

13 Steglich C, Futschik M E, Lindell D, et al. The challenge of regulation in a minimal photoautotroph: Non-coding RNAs in Prochlorococcus. PLoS Genet, 2008, 4: e1000173

14 Ionescu D, Voss B, Oren A, et al. Heterocyst-specific transcription of NsiR1, a non-coding RNA encoded in a tandem array of direct repeats in cyanobacteria. J Mol Biol, 2010, 398: 177-188

15 Ning D, Xu X. alr0117, a two-component histidine kinase gene, is involved in heterocyst development in Anabaena sp. PCC 7120. Microbiology, 2004, 150: 447-453

16 Elhai J, Wolk C P. Conjugal transfer of DNA to cyanobacteria. Methods Enzymol, 1988, 167: 747-754

17 Wang Y, Lechno-Yossef S, Gong Y, et al. Predicted glycosyl transferase genes located outside the HEP island are required for formation of heterocyst envelope polysaccharide in Anabaena sp. strain PCC 7120. J Bacteriol, 2007, 189: 5372-5378

18 Zhang W, Du Y, Khudyakov I, et al. A gene cluster that regulates both heterocyst differentiation and pattern formation in Anabaena sp. strain PCC7120. Mol Microbiol, 2007, 66: 1429-1443

19 Cai Y, Wolk C P. Use of a conditionally lethal gene in Anabaena sp. strain PCC 7120 to select for double recombinants and to entrap insertion sequences. J Bacteriol, 1990, 172: 3138-3145

$20 \mathrm{He} \mathrm{D}, \mathrm{Xu} \mathrm{X}$. CalA, a cyAbrB protein, binds to the upstream region of $\mathrm{fts} Z$ and is down-regulated in heterocysts in Anabaena sp. PCC 7120. Arch Microbiol, 2010, 192: 461-469

21 Argaman L, Hershberg R, Vogel J, et al. Novel small RNA-encoding genes in the intergenic regions of Escherichia coli. Curr Biol, 2001, 11: 941-950

22 Suno R, Niwa H, Tsuchiya D, et al. Structure of the whole cytosolic region of ATP-dependent protease FtsH. Mol Cell, 2006, 22: 575-585

23 Elhai J, Wolk C P. Developmental regulation and spatial pattern of expression of the structural genes for nitrogenase in the cyanobacterium Anabaena. EMBO J, 1990, 9: 3379-3388

24 Brantl S. Regulatory mechanisms employed by cis-encoded antisense RNAs. Curr Opin Microbiol, 2007, 10: 102-109

Open Access This article is distributed under the terms of the Creative Commons Attribution License which permits any use, distribution, and reproduction in any medium, provided the original author(s) and source are credited.

\section{Supporting Information}

Figure S1 The alignment of sequences of $f t s H$ genes in Anabaena 7120. Identical nucleotides are shaded in black. The afts $H$-encoding sequence in all3642 is indicated by a solid line.

Figure S2 The alignment of amino acid sequences of predicted FtsH proteins in Anabaena 7120. Identical amino acids are shaded in black and similar amino acids are shaded in gray. The region of All3642 used for production of anti-FtsH antiserum is indicated by a solid line. The Walker A motif, Walker B motif and $\mathrm{Zn}^{2+}$-metalloprotease active site are denoted in green, yellow and red respectively.

The supporting information is available online at csb.scichina.com and www.springerlink.com. The supporting materials are published as submitted, without typesetting or editing. The responsibility for scientific accuracy and content remains entirely with the authors. 